\title{
ON THE BENDING OF A CLAMPED PLATE*
}

\section{By A. WEINSTEIN (University of Toronto) and D. H. ROCK (Rhode Island State College)}

The present paper contains an application of a recently developed variational method $^{1}$ to the boundary value problem of the bending of a clamped plate of arbitrary shape. It will be shown that this problem can be linked to the simpler problem of the equilibrium of a membrane by a chain of intermediate problems, which can be solved explicitly and in finite form in terms of the membrane problem. In the intermediate problems, the deflection converges uniformly in the domain of the plate (including the boundary) to the deflection of the clamped plate, and the derivatives of all orders of the deflection converge uniformly in every domain completely interior to the plate. (In the Ritz method, not even the convergence of the slopes can be guaranteed. ${ }^{2}$ ) The method yields numerical results for plates of all shapes for which the membrane problem (which we shall call the base problem) admits an explicit solution. As an example we shall consider a clamped square plate under a uniform load. This problem has been the object of numerous investigations, ${ }^{3}$ some of which are theoretical, while others are purely numerical, use infinite simple and double series, and operate with an infinite number of linear equations and an infinite number of unknowns. ${ }^{4}$ An inspection of the general formulae derived in the present paper, formulae which become simple in numerical applications, would show how some of the numerical methods might be rendered rigorous. ${ }^{5}$ The convergence of higher derivatives is of great practical interest for the approximate computation of the stresses. (Cf. Handbuch der Physik, Springer, Berlin, Vol. VI, 1928, pp. 220-221.)

Let us denote the domain of a clamped plate by $S$ and its boundary by $C$. The deflection $w(x, y)$ corresponding to a load $q(x, y)$ and to a flexural rigidity $D$ satisfies the differential equation

$$
\Delta \Delta w=q / D
$$

with the boundary conditions

$$
w=0 \text {, }
$$

$$
d w / d n=0,
$$

on $C$. It is well known that $w$ is the solution of the variational problem $V P$,

$$
J(w) \equiv \iint_{S}\left[(\Delta w)^{2}-2 \bar{q} w\right] d x d y=\min .
$$

* Received Feb. 8, 1944.

1 A. Weinstein, Mémorial des Sciences Mathématiques, No. 88, 1937.

2 Cf. R. Courant, Variational methods for the solutions of problems of equilibrium and vibrations, Bull. Amer. Math. Soc., 49, 1-23, 1943, especially p. 11. See also K. Friedrichs, Math. Annalen, 98, 217, 1928. The method of finite differences does not give satisfactory numerical results for clamped plates.

${ }^{3}$ The bibliography given in S. Timoshenko, Plates and shells, McGraw-Hill, 1940, p. 222 covers papers from 1902 to 1939 and shows the persistent interest in this problem.

H. W. March, Trans. Amer. Math. Soc., 27, 307-317, 1925, proves the weak convergence of the approximations given by his series. Cf. I. S. Sokolnikoff, Mathematical theory of elasticity, Brown University, 1941, p. 387.

' Cf. for instance a note by C. Miranda, Rend. Semin. Mat. di Roma, 1, 262-266, 1937. 
where $\bar{q}=q / D$ and the boundary conditions (2) and (3) are in effect. By withdrawing the condition $d w / d n=0$, we obtain the variational base problem $V P_{0}: J\left(w_{0}\right)=\min$., with the condition $w_{0}=0$ on $C$. The corresponding differential equations problem $D P_{0}$ is: $\Delta \Delta w_{0}=\bar{q}$, with the boundary conditions $w_{0}=0$ and $\Delta w_{0}=0$ on $C$, the latter condition being a natural boundary condition which is automatically satisfied by a solution of $V P_{0}$. It is well known that $D P_{0}$ can be solved in terms of the problem of the equilibrium of a membrane. In fact, putting $\Delta w_{0}=f_{0}$ in $D P_{0}\left(w_{0}=0\right.$ on $\left.C\right)$, we have $\Delta f_{0}=\bar{q}$ in the domain $S$ and $f_{0}=0$ on $C \cdot{ }^{6}$ Let us denote the solution of the equation $\Delta w_{0}=f_{0}$ with the boundary condition $w_{0}=0$ by $w_{0}=G f_{0}$. Thus we have $f_{0}=G \bar{q}$, whence $w_{0}=G G \bar{q}$. (The formula $w_{0}=G f_{0}$ can be written explicitly in the form

$$
w_{\theta}=-\iint_{S} g(x, y, \xi, \eta) f_{0}(\xi, \eta) d \xi d \eta,
$$

where $g(x, y, \xi, \eta)$ is the Green's function for the domain $S$.)

We now link $V P_{0}$ to $V P$ by a chain of intermediate variational problems $V P_{1}, V P_{2}, \cdots$ introduced in the following way. We let $p_{1}(x, y), p_{2}(x, y), \cdots$ denote a complete (but not necessarily orthogonal) sequence of linearly independent harmonic functions in $S$. (It has been shown, l.c., ${ }^{1}$ how a sequence of this kind can be derived from the solutions of the problem of a vibrating membrane for any domain $S$.)

$V P_{m}(m=1,2, \cdots)$ is then defined as the problem of finding the solution $w_{m}$ of $J\left(w_{m}\right)=$ min., with the boundary conditions

$$
w_{m}=0 \text { on } C, \quad \int_{c} p_{k} \frac{d w_{m}}{d n} d s=0, \quad k=1,2, \cdots, m .
$$

By Green's formula these conditions can be replaced by the conditions

$$
w_{m}=0 \text { on } C, \quad\left(p_{k}, \Delta w_{m}\right)=0, \quad k=1,2, \cdots, m,
$$

where

$$
\left(p_{k}, \Delta w_{m}\right)=\iint_{S} p_{k} \Delta w_{m} d x d y .
$$

(Similar notations for the "scalar product" of two function, like $p_{k}$ and $\Delta w_{m}$, will be used throughout this paper.) The corresponding differential problem $D P_{m}$ is given by the differential equation

$$
\Delta \Delta w_{m}=\bar{q}
$$

with the conditions (4) and the natural boundary condition

$$
\Delta w_{m}=a_{m 1} p_{1}+a_{m 2} p_{2}+\cdots+a_{m m} p_{m} \text { on } C,
$$

where $a_{m 1}, a_{m 2}, \cdots, a_{m m}$ are constants to be determined. The solutions of $D P_{m}$ can be easily obtained in terms of solutions of the membrane problem already used to solve $D P_{0}$. In fact, putting $\Delta w_{m}=f_{m}\left(w_{m}=0\right.$ on $\left.C\right)$, we have, in our notation, $w_{m}=G f_{m}$. Also, we obtain from (5),

$$
\Delta f_{m}=\bar{q},
$$

- For rectangular plates $D P_{0}$ is the problem of the supported plate. 
and from the boundary condition (6),

$$
f_{m}=a_{m 1} p_{1}+a_{m 2} p_{2}+\cdots+a_{m m} p_{m} .
$$

These can be written as follows:

$$
\begin{gathered}
\Delta\left(f_{m}-\sum_{i=1}^{m} a_{m i} p_{i}\right)=\bar{q} \text { in } S . \\
f_{m}-\sum_{i=1}^{m} a_{m i} p_{i}=0 \text { on } C .
\end{gathered}
$$

Therefore we have in $S$

$$
f_{m}-\sum_{i=1}^{m} a_{m i} p_{i}=G \bar{q},
$$

and since $w_{m}=G f_{m}$, it follows that

$$
w_{m}=G G \bar{q}+\sum_{i=1}^{m} a_{m i} G p_{i}
$$

where $G G \bar{q}=w_{0}$ is the solution of the base problem.

The conditions $\left(p_{k}, \Delta w_{m}\right)=0$ yield, in view of (9), the following system of $m$ linear equations for the $m$ constants $a_{m 1}, a_{m 2}, \cdots, a_{m m}$ :

$$
\sum_{i=1}^{m} a_{m i}\left(p_{i}, p_{k}\right)=-\left(\bar{q}, G p_{k}\right), \quad k=1,2, \cdots, m,
$$

which can be solved, since their determinant is Gram's determinant of the independent functions $p_{1}, p_{2}, \cdots, p_{m}$, and is different from zero.

In another paper, based on a previously developed method for the computation of frequencies and buckling loads, ${ }^{7}$ it will be proved that the approximate solutions $w_{m}$ and their first derivatives converge to the deflection and slopes of the clamped plate. Here we shall apply our formulae to the case of a uniformly loaded square plate. The domain $S$ will be defined by the inequalities $|x| \leqq \pi / 2,|y| \leqq \pi / 2$. We put $\bar{q}=q / D=1$. Since the deflection of a uniformly loaded square plate is symmetrical with respect to the coordinate axes, we may use a sequence of even harmonic functions $p_{i}(x, y)$ as given by (12) below. ${ }^{8}$ All computations can be performed without the use of Green's function for the square. The deflection $w_{0}$ of the supported plate is given by the well known formulae of Navier.

Calculation of $w_{m}$ for the uniformly loaded square plate.

We use the set of functions

$$
p_{i}(x, y)=\frac{\cosh \alpha_{i} x \cos \alpha_{i} y+\cos \alpha_{i} x \cosh \alpha_{i} y}{\cosh \left(\alpha_{i} \pi / 2\right)}, \quad\left(\alpha_{i}=2 i-1\right),
$$

7 N. Aronszajn and A. Weinstein, On the unified theory of eigen-values of plates and membranes, Amer. J. Math., 64, 625-645, 1942.

${ }^{8}$ For non-uniform loading, the sequence given $1 . c .1, ~ \ 16$ must be used. 
and denote $G p_{i}$ by $v_{i}$. Then, by the definition of $G$, we have $\Delta v_{i}=\Delta G p_{i}=p_{i}$ in $S$, with $v_{i}=0$ on $C$.

If we set $v_{i}=u_{1}+u_{2}$, where

$$
u_{1}=X(x) \frac{\cos \alpha_{i} y}{\cosh \left(\alpha_{i} \pi / 2\right)}, \quad u_{2}=Y(y) \frac{\cos \alpha_{i} x}{\cosh \left(\alpha_{i} \pi / 2\right)},
$$

then $X(x)$ and $Y(y)$ satisfy the differential equations

$$
X^{\prime \prime}-\alpha_{i}^{2} X=\cosh \alpha_{i} x, \quad Y^{\prime \prime}-\alpha_{i}^{2} Y=\cosh \alpha_{i} y,
$$

with the boundary conditions

$$
X( \pm \pi / 2)=0, \quad Y( \pm \pi / 2)=0 .
$$

The general solution for $X(x)$ is

$$
X(x)=\frac{1}{2 \alpha_{i}} x \sinh \alpha_{i} x+A \cosh \alpha_{i} x+B \sinh \alpha_{i} x,
$$

where $A$ and $B$ are determined by the boundary conditions. Hence

$$
X=\frac{1}{2 \alpha_{i}}\left[x \sinh \alpha_{i} x-\frac{\pi}{2} \tanh \frac{1}{2} \alpha_{i} \pi \cosh \alpha_{i} x\right] .
$$

The solution for $Y(y)$ is given by a similar expression, so finally

$$
\begin{aligned}
v_{i}=\frac{1}{2 \alpha_{i} \cosh \left(\alpha_{i} \pi / 2\right)}\left\{\left[x \sinh \alpha_{i} x-\frac{\pi}{2} \tanh \frac{1}{2} \alpha_{i} \pi \cosh \alpha_{i} x\right] \cos \alpha_{i} y\right. \\
\left.+\left[y \sinh \alpha_{i} y-\frac{\pi}{2} \tanh \frac{1}{2} \alpha_{i} \pi \cosh \alpha_{i} y\right] \cos \alpha_{i} x\right\} .
\end{aligned}
$$

Using (13), we obtain the general formula for $\left(\bar{q}, G p_{i}\right)=\left(1, v_{i}\right)$,

$$
\left(1, v_{i}\right)=\frac{4 \sin \left(\alpha_{i} \pi / 2\right)}{\alpha^{3}}\left[\frac{\pi}{2} \operatorname{sech}^{2} \frac{1}{2} \alpha_{i} \pi-\frac{1}{\alpha_{i}} \tanh \frac{1}{2} \alpha_{i} \pi\right] .
$$

For $\left(p_{i}, p_{k}\right)$ we have

$$
\begin{aligned}
\left(p_{i}, p_{k}\right) & =\frac{8 \alpha_{i} \alpha_{k}(-)^{i+k}}{\left(\alpha_{i}^{2}+\alpha_{k}^{2}\right)^{2}}, \quad i \neq k, \\
& =\pi\left[\frac{\pi}{2} \operatorname{sech}^{2} \frac{1}{2} \alpha_{i} \pi+\frac{1}{\alpha_{i}} \tanh \frac{1}{2} \alpha_{i} \pi\right]+\frac{2}{\alpha_{i}^{2}}, \quad i=k .
\end{aligned}
$$

From (14) we find that

$$
\begin{gathered}
\left(1, v_{1}\right)=-2.670644, \quad\left(1, v_{2}\right)=0.049299, \quad\left(1, v_{3}\right)=-0.006400, \\
\left(1, v_{4}\right)=0.001666, \text { etc. }
\end{gathered}
$$

and from (15) we get the following table for $\left(p_{i}, p_{k}\right),(i, k=1,2,3,4)$ : 


\begin{tabular}{|c|c|c|c|c|}
\hline$i \quad k$ & 1 & 2 & 3 & 4 \\
\hline 1 & 5.665118 & -0.240000 & 0,059172 & -0.022400 \\
\hline 2 & & 1.270836 & -0.103806 & 0.049941 \\
\hline 3 & & & 0.708321 & -0.051132 \\
\hline 4 & & & & 0.489615 \\
\hline
\end{tabular}

The equations for the determination of $a_{m i}$ are then

$$
\left.\begin{array}{rl}
5.665118 a_{11} & =2.670644, \text { for } a_{11}, \\
5.665118 a_{21}-0.240000 a_{22} & =2.670644 \\
-0.240000 a_{21}+1.270836 a_{22} & =-0.049299
\end{array}\right\} \text { for } a_{21} \text { and } a_{22},
$$

These yield the successive values

$$
\begin{array}{ll}
a_{11}=0.471419, & \\
a_{21}=0.473564, & a_{22}=0.050641,
\end{array}
$$

Then, since the solution for the simply supported plate is given by

$$
w_{0}(x, y)=\frac{16}{\pi^{2}} \sum_{m} \sum_{n} \frac{\sin m(x+\pi / 2) \sin n(y+\pi / 2)}{m n\left(m^{2}+n^{2}\right)^{2}}, \quad(m, n=1,3,5, \cdots),
$$

the successive approximations to the deflection are:

$$
\begin{aligned}
& w_{1}=w_{0}+0.471419 v_{1}, \\
& w_{2}=w_{0}+0.473564 v_{1}+0.050641 v_{2},
\end{aligned}
$$

The maximum deflection, which occurs at the center of the plate, is found to be 0.123342 when $w_{2}$ is used. The next approximation affects only the fourth significant figure.

A calculation of the normal derivative of $w_{m}(m=0,1,2,3,4)$ along $x=\pi / 2$ for values of $y$ from 0 to $\pi / 2$ at intervals of $\pi / 16$ yields:

\begin{tabular}{c|c|c|c|c|c|c|c|c|c}
$m$ & $y=0$ & $\pi / 16$ & $2 \pi / 16$ & $3 \pi / 16$ & $4 \pi / 16$ & $5 \pi / 16$ & $6 \pi / 16$ & $7 \pi / 16$ & $\pi / 2$ \\
\hline 0 & -.41795 & -.41087 & -.08954 & -.35409 & -.30519 & -.24382 & -.17095 & -.08839 & 0 \\
1 & -.00763 & -.00686 & -.00445 & -.00062 & +.00443 & +.00912 & +.01221 & +.01100 & 0 \\
2 & +.00197 & +.00122 & -.00047 & -.00189 & -.00208 & -.00060 & +.00216 & +.00355 & 0 \\
3 & -.00053 & -.00019 & +.00044 & +.00055 & -.00020 & -.00093 & -.00018 & +.00151 & 0 \\
4 & +.00029 & -.00003 & -.00031 & +.00008 & +.00036 & -.00021 & -.00033 & +.00066 & 0
\end{tabular}

The maximum value of the slope in the interior of the plate is found to be about -0.122 ; this occurs at $x=5 \pi / 16, y=0$. A comparison with the maximum deviation of the normal derivative along the edge for $m=4$ shows that the latter is less than $1 \%$ of the maximum slope in the interior of the plate. 